\title{
Pulse Response of a Kinetic Inductance Detector in the Nonlinear Regime
}

\author{
Chiara Bellenghi ${ }^{1,2}$ D $\cdot$ L. Cardani ${ }^{2} \cdot$ N. Casali ${ }^{2} \cdot$ I. Colantoni $^{2,3} \cdot$ A. Cruciani $^{2}$. \\ G. Pettinari ${ }^{4} \cdot$ M. Vignati $^{2}$
}

Published online: 19 March 2020

๑) Springer Science+Business Media, LLC, part of Springer Nature 2020

\begin{abstract}
Over the last few years, kinetic inductance detectors (KIDs) became the object of increasing interest as photon and phonon detectors. From this perspective, the pulse response of such detectors deserves an in-depth study. In most applications, the sensitivity of the KID is ultimately limited by the white noise from the cryogenic amplifier, which is reduced by increasing the power supplied to the device. On the other hand, a high readout power leads to a nonlinear response of the microresonator, originating from the dependence on the current acquired by the kinetic inductance. This paper describes a model for the response to optical pulses of a KID driven to the nonlinear regime, taking into account not only the electrical effects but also the thermal ones induced by power absorption. The model has been validated on data collected using an aluminium resonator developed within the CALDER project (http://www.roma1.infn.it/exp/calder/).
\end{abstract}

Keywords Microwave kinetic inductance detectors · Kinetic inductance nonlinearity $\cdot$ Thermal effects

Chiara Bellenghi

chiara.bellenghi@tum.de

1 Dipartimento di Fisica, Sapienza Università di Roma, 00185 Rome, Italy

2 INFN Sezione di Roma, Rome, Italy

3 Consiglio Nazionale delle Ricerche, Istituto di Nanotecnologia (CNR - NANOTEC), c/o Dip. Fisica, Sapienza Università di Roma, 00185 Rome, Italy

4 Consiglio Nazionale delle Ricerche, Istituto di Fotonica e Nanotecnologie (CNR - IFN), Via Cineto Romano 42, 00156 Rome, Italy 


\section{Introduction}

A model of the KID response in the high-power nonlinear regime has already been proposed and demonstrated at Caltech for an MKID under continuous optical illumination [1]. The transmission past a KID resonator can be written as [2]:

$$
S_{21}=1-\frac{Q}{Q_{\mathrm{c}}} \frac{1}{1+2 j y}
$$

where $Q_{\mathrm{c}}$ is the coupling quality factor, $\mathrm{Q}$ is the total quality factor of the resonator, given by $Q^{-1}=Q_{\mathrm{c}}^{-1}+Q_{i}^{-1}$ with $Q_{i}$ the internal quality factor, and y is the generator detuning from the resonant frequency $\left(f_{\mathrm{r}}\right)$ measured in linewidths which in the lowpower regime is just $y_{0}=Q\left(f-f_{\mathrm{r}}\right) / f_{\mathrm{r}}$. When moving to the high-power nonlinear regime, the increase in the kinetic inductance produces a shift of the resonant frequency towards lower frequencies. To account for this effect, the detuning becomes $y=y_{0}+a /\left(1+4 y^{2}\right)$, where $a=\left(Q^{3} P_{g}\right) /\left(Q_{\mathrm{c}} \pi f_{\mathrm{r}} E_{*}\right)$ is the nonlinearity parameter, which is proportional to the cube of total quality factor $Q$ and the readout power $P_{g}$ and depends on $E_{*}$, i.e. the nonlinearity scaling energy. In practice, the resonant frequency moves from $y_{0}=0$ to $y=0$, that is $y_{0}=-a$.

As regards the resonator response, contrary to what happens in the low-power linear regime [2], in the nonlinear framework the phase $(\delta \phi)$ and amplitude $(\delta A)$ components of the signal are correlated: In particular, the dissipative (amplitude) response enters the reactive (phase) one ${ }^{1}$ :

$$
\delta \phi(y)=\frac{\delta \phi_{0}}{1+4 y^{2}} \frac{1-\frac{4 a}{\left(1+4 y^{2}\right)^{2}} \frac{\delta A_{0}}{\delta \phi_{0}}}{1+\frac{8 a y}{\left(1+4 y^{2}\right)^{2}}} .
$$

Here, the $1 /\left(1+4 y^{2}\right)$ is to account for the detuning of the generator tone from the resonant frequency and $\delta \phi_{0}$ and $\delta A_{0}$ are the two signals measured on resonance $(y=0)$ when operating the KID in the low-power linear regime.

Contrary to what has been demonstrated in Ref. [1], we found out that we were affected not only by electrical effects but also by thermal ones caused by an increased quasiparticle effective temperature due to power absorption [3]. This temperature variation contributes to the magnitude of both the phase and amplitude signals. Moreover, the thermal effects introduce an additional shift of the resonant frequency in the resonator response, which from an operational point of view is undistinguishable from the one induced by the kinetic inductance nonlinearity. Thus, the two effects need to be disentangled in the model.

To extract the temperature of the KID, we exploited the quasiparticle lifetime, whose dependency on the temperature is well known thanks to the BCS nature of the aluminium superconductor [4]. In the following, all the considerations made to

\footnotetext{
${ }^{1}$ Eq. 2 is derived by converting in phase variation the frequency variation that can be found in Ref. [1]
} 
Table 1 The best fit parameters for an aluminium MKID biased with a readout power of $-92 \mathrm{dBm}$, corresponding to a null nonlinearity parameter, and with $-63 \mathrm{dBm}$, corresponding to $a=0.46$

\begin{tabular}{lll}
\hline & \multicolumn{2}{l}{ Resonance parameters } \\
\cline { 2 - 3 } & Low power & High power \\
\hline$P_{\text {in }}$ & $-92.1 \mathrm{dBm}$ & $-62.6 \mathrm{dBm}$ \\
$a$ & 0.00 & 0.46 \\
$f_{\mathrm{r}}$ & $2556.370 \mathrm{MHz}$ & $2556.368 \mathrm{MHz}$ \\
$Q$ & $93 \times 10^{3}$ & $109 \times 10^{3}$ \\
$Q_{\mathrm{c}}$ & $109 \times 10^{3}$ & $109 \times 10^{3}$ \\
$Q_{\mathrm{i}}$ & $627 \times 10^{3}$ & inf \\
\hline
\end{tabular}

In the high-power regime, we are no longer sensitive to the increase in the internal quality factor and the total $Q$ equals the coupling one
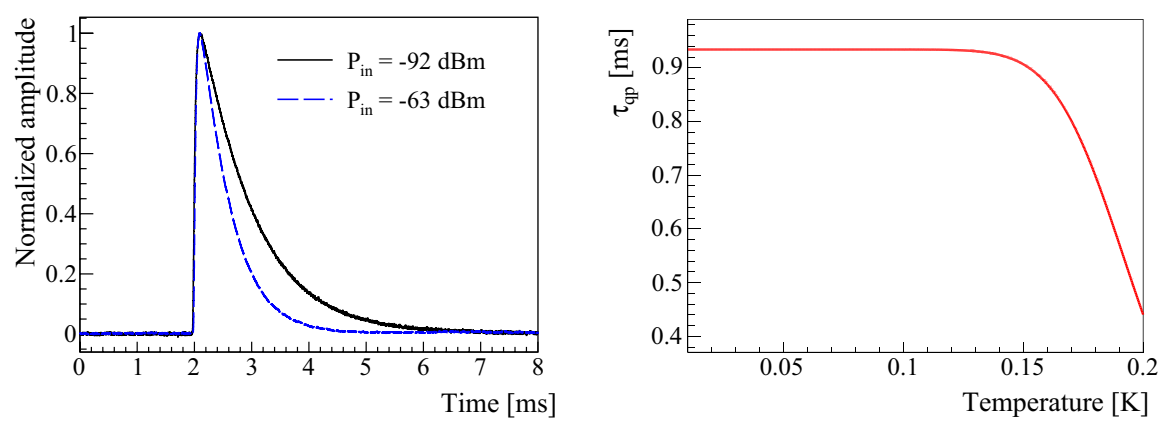

Fig. 1 Left: Phase pulses acquired on resonance $(y=0)$ in the low-power (solid black) and in the highpower regime (dashed blue). Both the amplitudes are scaled to 1 . Moving from the low- to the highpower regime, a reduction in the pulse decay time occurs. Right: Quasiparticle lifetime as a function of the temperature for aluminium (Color figure online)

finalize the pulse response model will be reported, together with all the methods adopted to get the quantities needed to validate it on data collected using a 60-nmthick Al KID with $4 \mathrm{~mm}^{2}$ active area, deposited on a $2 \times 2 \mathrm{~cm}^{2}, 300-\mu \mathrm{m}$-thick Si substrate [5]. This sensor has a measured fraction of kinetic inductance to the total inductance $\alpha=2.54 \%$, and its transition temperature is $T_{\mathrm{c}}=1.18 \mathrm{~K}$, corresponding to a superconductive gap $\Delta_{0}=179 \mu \mathrm{eV}$. The resonance parameters were derived by fitting the complex transmission $S_{21}$ measured in a frequency sweep around the resonance using the model described in Ref. [6], and they are all reported in Table 1.

\section{Evidence and Effects of Temperature Variations}

Moving from the low- to the high-power regime, we observed a decrease in the pulses decay time, shown in the left panel of Fig. 1. This time constant is identified with the quasiparticle lifetime $\tau_{\mathrm{qp}}$ [7], whose decreasing trend as a function of the temperature is known from the BCS theory of superconductivity [4], which 

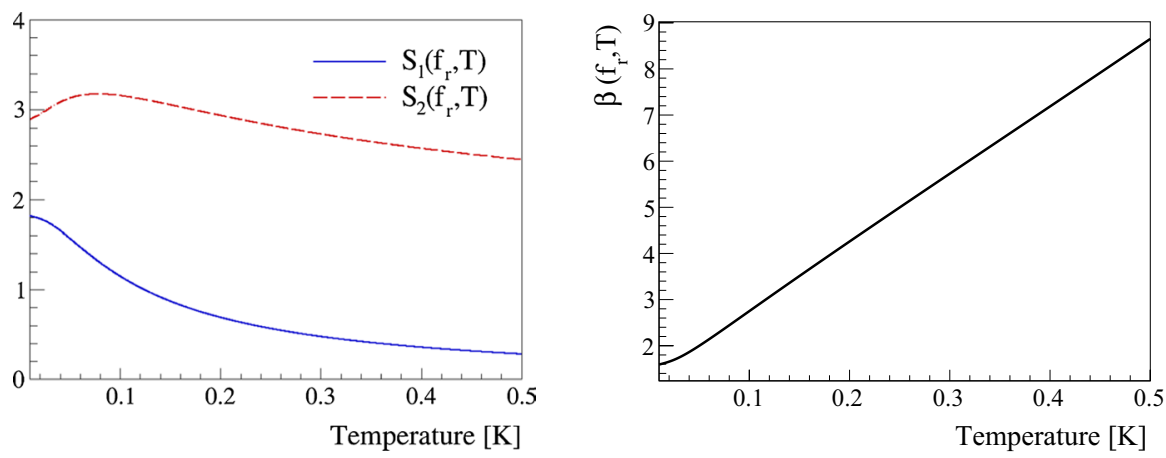

Fig. 2 Left: Dimensionless functions of the frequency and effective temperature to which the amplitude and phase responses are, respectively, proportional, $S_{1}\left(f_{\mathrm{r}}, T\right)$ (solid blue) and $S_{2}\left(f_{\mathrm{r}}, T\right)$ (dashed red). Right: $\beta\left(f_{\mathrm{r}}, T\right)=S_{2}\left(f_{\mathrm{r}}, T\right) / S_{1}\left(f_{\mathrm{r}}, T\right)$ trend as a function of the temperature (Color figure online)

can fairly be applied to our aluminium microresonator as long as the experimentally observed deviation from the BCS exponential trend at low temperatures [8] is taken into account (Fig. 1 right):

$$
\frac{1}{\tau_{\mathrm{qp}}(T)}=\frac{1}{\tau_{\max }}+\frac{1}{\tau_{0}} \sqrt{\pi}\left(\frac{2 \Delta}{k_{\mathrm{B}} T_{\mathrm{c}}}\right)^{5 / 2} \sqrt{\frac{T}{T_{\mathrm{c}}}} e^{\frac{-\Delta}{k_{\mathrm{B}} T}} .
$$

Here, $\tau_{\max }$ is the maximum quasiparticle lifetime value reached at low temperatures and $t_{0}$ is a material-specific electron-phonon scattering time. Therefore, we use the quasiparticle recombination time as a proxy for the quasiparticle effective temperature. The temperature increase causes the resonant frequency to shift towards lower frequencies according to formula [9]:

$$
\frac{\delta f_{r}}{f_{r}}=-\frac{\alpha}{2} S_{2}\left(f_{\mathrm{r}}, T\right) \sqrt{\frac{2 \pi k_{B} T}{\Delta(T)}} e^{\frac{-\Delta(T)}{k_{B} T}},
$$

where $S_{2}\left(f_{\mathrm{r}}, T\right)$ is a dimensionless function describing the phase response to temperature changes.

The increased temperature also impacts on the responsivity, which, according to the BCS theory, can be parameterized in terms of $S_{2}\left(f_{\mathrm{r}}, T\right)$ and $S_{1}\left(f_{\mathrm{r}}, T\right)$ (another dimensionless function of the frequency and effective temperature), to which the phase $\left(\delta \phi_{0}\right)$ and amplitude $\left(\delta A_{0}\right)$ signals are, respectively, proportional and whose trend is shown in Fig. 2 left.

The low-power signals need to be corrected to take into account the temperature variation; therefore, they are substituted with $\delta \phi_{0}\left(S_{2}\left(f_{\mathrm{r}}, T\right) / S_{2}\left(f_{\mathrm{r}}, T_{0}\right)\right)$ and $\delta A_{0}$ $\left(S_{1}\left(f_{\mathrm{r}}, T\right) / S_{1}\left(f_{\mathrm{r}}, T_{0}\right)\right)$, where $T_{0}$ is the low-power effective temperature. Including these corrections and recalling that $\delta \phi_{0} / \delta A_{0}=S_{2}\left(f_{\mathrm{r}}, T_{0}\right) / S_{1}\left(f_{\mathrm{r}}, T_{0}\right)$ [10] and that $\beta\left(f_{\mathrm{r}}, T\right)=S_{2}\left(f_{\mathrm{r}}, T\right) / S_{1}\left(f_{\mathrm{r}}, T\right)$ (Fig. 2 right), the phase response of the KID with temperature can be written as: 


$$
\delta \phi(y)=\frac{S_{2}\left(f_{\mathrm{r}}, T\right)}{S_{2}\left(f_{\mathrm{r}}, T_{0}\right)} \frac{\delta \phi_{0}}{1+4 y^{2}} \frac{1-\frac{4 a}{\left(1+4 y^{2}\right)^{2}} \frac{1}{\beta\left(f_{\mathrm{r}}, T\right)}}{1+\frac{8 a y}{\left(1+4 y^{2}\right)^{2}}}
$$

\section{Probing the Pulse Response Model}

To probe the new response model, the aluminium MKID was tested. We performed a fit of the transmission to extract the fundamental parameters of the resonator [6]. To evaluate the low-power signal, we biased the resonator with a readout power of - $92 \mathrm{dBm}$ and performed a frequency scan measuring the resonator response to optical pulses (bursts of photons) at different frequencies moving along the resonance. The chosen readout power was low enough to have $a=0$ (cfr. Table 1).

The signal is measured as the magnitude of the pulse obtained averaging all the acquired pulses, evaluated exploiting the signals produced by the optical system (a room-temperature LED and an optical fibre that drives the optical pulses). These light signals are faster than the ring-down response of the resonator, $\tau_{\text {ring }}=Q / \pi f_{\mathrm{r}} \sim 10 \mu s$ [11]. In particular, each pulse is acquired for the real (I) and imaginary (Q) parts of the resonator transmission $\left(S_{21}\right)$ and then converted into changes in phase $\delta \phi$ and amplitude $\delta A$ relative to the centre of the resonance loop. Pulses were collected moving from $y \simeq-0.8$ to $y \simeq+0.8$ (corresponding to $\pm 20 \mathrm{kHz}$ around $f_{\mathrm{r}}$ ). As expected for the linear regime, the two components were completely uncorrelated and symmetric with respect to the resonant frequency, where they reach their maxima $\delta \phi_{0}$ and $\delta A_{0}$, according to the relations $\delta \phi=\delta \phi_{0} /\left(1+4 y_{0}^{2}\right)$ and $\delta A=\delta A_{0} /\left(1+4 y_{0}^{2}\right)$.

A frequency scan was then performed biasing the chip with $-63 \mathrm{dBm}$. Since the phase signal was $\sim$ six times larger than the amplitude one, it is the one used in the analysis. The effective temperature in the high-power regime at the various bias frequencies (Fig. 3 left) was evaluated via the measurement of the pulses decay time. The pulses analysis showed that the maximum effective temperature is reached on resonance $(y=0)$, where the power absorption is maximum as well. The retrieved temperature was $198 \mathrm{mK}$, corresponding to a resonant frequency shift of $-2 \mathrm{kHz}$ (see Fig. 3 right), evaluated from Eq. (4) using our resonant frequency of $\sim 2.5 \mathrm{GHz}$. By including this shift in the resonance fit function, we found a nonlinearity parameter equal to 0.46 (cfr. Table 1). Without the thermal correction, the fit returned instead $a=0.56$, and the obtained reduction is compatible with $Q \delta f_{\mathrm{r}} / f_{\mathrm{r}} \sim-0.1$ using the merit factor $Q=109 \times 10^{3}$ returned by the same high-power resonance fit.

Putting all the needed quantities in Eq. (5), we then applied it to the experimental data and the results are shown in the left panel of Fig. 4. As can be observed, the temperature-independent model proposed in Ref. [1] (red squares in the figure) does not reproduce the data, while the new one including thermal effects (green triangles in the figure) does rather well. Considering the maximum deviation of $10 \%$ between 

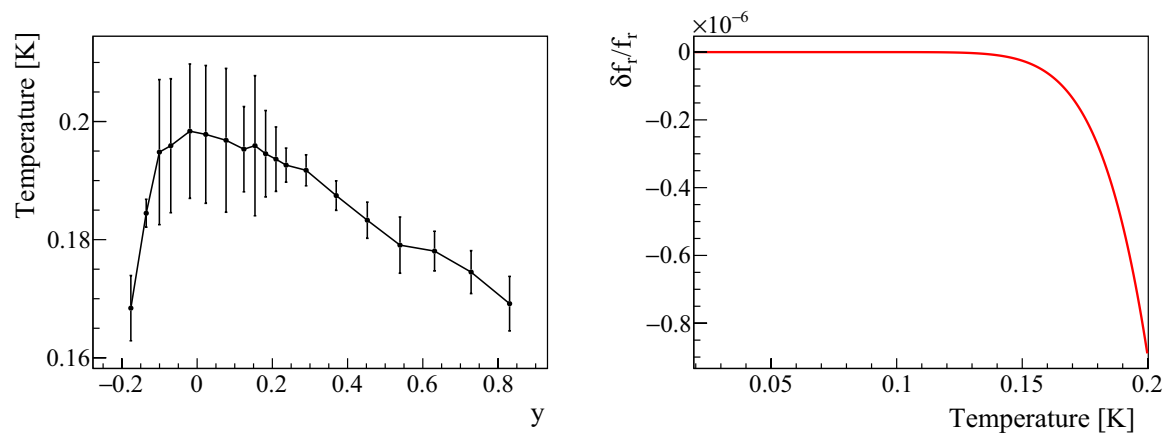

Fig. 3 Left: Effective quasiparticle temperature evaluated from the measured $\tau_{\mathrm{qp}}$. The maximum heating is reached on resonance $(y=0)$, where the power absorption is likewise maximum. Right: Resonant frequency shift towards lower frequencies due to thermally generated quasiparticles. With $f_{\mathrm{r}} \sim$ $2.5 \mathrm{GHz}$, for $\mathrm{T} \sim 200 \mathrm{mK}$, a shift of $\sim 2 \mathrm{kHz}$ is foreseen. We recall here that for this resonator $\alpha=2.5 \%$ and $\Delta_{0}=179 \mu \mathrm{eV}$ (Color figure online)

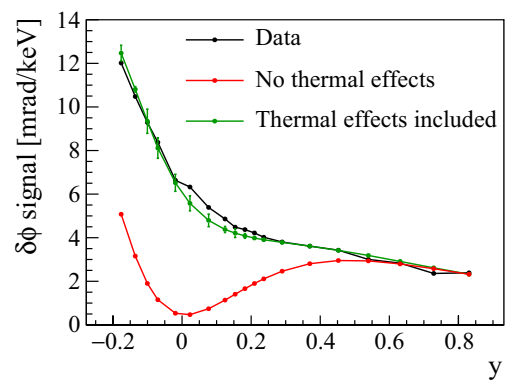

Fig. 4 The new pulse response model (green triangles) is superimposed to the experimental data (black circles). Signals acquired varying the bias frequency. The model proposed in [1] (red squares) does not reproduce the data, while the one including thermal effects demonstrates to cover them within a maximum deviation of $10 \%$ (Color figure online)

it and the experimental observation, we got a satisfactory understanding of the KID pulse response.

\section{Conclusions}

We developed a response model for a KID illuminated with optical pulses and operated in the nonlinear regime of response. By including the thermal effects due to power absorption, consisting in an additional frequency shift towards lower frequency and in an extra contribution to the magnitude of the signal, we were able to reproduce the experimental data deviating from them by a $10 \%$ at most.

Acknowledgements We thank Loren J. Swenson for useful discussions on the kinetic inductance behaviour. This work was supported by the European Research Council (FP7/2007-2013) under Contract CALDER No. 335359. 


\section{References}

1. L.J. Swenson, P.K. Day, B.H. Eom, H.G. Leduc, N. Llombart, C.M. McKenney, O. Noroozian, J. Zmuidzinas, J. Appl. Phys. 113, 104501 (2013). https://doi.org/10.1063/1.4794808

2. J. Zmuidzinas, Annu. Rev. Condens. Matter Phys. 3, 52-92 (2012). https://doi.org/10.1146/annur ev-conmatphys-020911-125022

3. P.J. de Visser, S. Withington, D.J. Goldie, J. Appl. Phys. 108(11), 114504 (2010). https://doi. org/10.1063/1.3517152

4. S.B. Kaplan, C.C. Chi, D.N. Langenberg, J.J. Chang, S. Jafarey, D.J. Scalapino, Phys. Rev. B 14, 4854 (1976). https://doi.org/10.1103/PhysRevB.14.4854

5. L. Cardani, N. Casali, I. Colantoni, A. Cruciani, F. Bellini, M.G. Castellano, C. Cosmelli, A. D’Addabbo, S. Di Domizio, M. Martinez, C. Tomei, M. Vignati, Appl. Phys. Lett. 110, 033504 (2017). https://doi.org/10.1063/1.4974082

6. N. Casali, F. Bellini, L. Cardani, M.G. Castellano, I. Colantoni, A. Coppolecchia, C. Cosmelli, A. Cruciani, A. D’Addabbo, S. Di Domizio, M. Martinez, C. Tomei, M. Vignati, J. Low Temp. Phys. 184, 142-147 (2016). https://doi.org/10.1007/s10909-015-1358-y

7. L. Cardani, I. Colantoni, A. Cruciani, S. Di Domizio, M. Vignati, F. Bellini, N. Casali, M.G. Castellano, A. Coppolecchia, C. Cosmelli, C. Tomei, Appl. Phys. Lett. 107, 093508 (2015). https://doi. org/10.1063/1.4929977

8. R. Barends, J.J.A. Baselmans, S.J.C. Yates, J.R. Gao, J.N. Hovenier, T.M. Kaplapwijk, Phys. Rev. Lett. 100, 257002 (2008). https://doi.org/10.1103/PhysRevLett.100.257002

9. J. Gao, J. Zmuidzinas, B.A. Mazin, P.K. Day, H.G. Leduc, Nucl. Instr. Meth. Phys. Res. A 559, 799-801 (2006). https://doi.org/10.1016/j.nima.2005.12.075

10. J. Gao, The Physics of Superconducting Microwave Resonators, PhD thesis, California Institute of Technology (2008)

11. M. Vignati, F. Bellini, L. Cardani, N. Casali, M.G. Castellano, I. Colantoni, A. Coppolecchia, C. Cosmelli, A. Cruciani, A. D’Addabbo, S. Di Domizio, M. Martinez, C. Tomei, Eur. Phys. J. C 75, 353 (2015). https://doi.org/10.1140/epjc/s10052-015-3575-6

Publisher's Note Springer Nature remains neutral with regard to jurisdictional claims in published maps and institutional affiliations. 